\title{
Pheochromocytoma Manifestation in Cardiovascular System: A Comprehensive Explanatory Review
}

\author{
Rizki Amalia ${ }^{a^{*}}$, Budi Susetyo Pikir ${ }^{\mathrm{b}}$ \\ a rizkiamalia0291@gmail.com

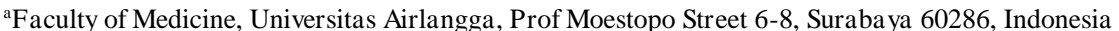 \\ ${ }^{b}$ Department of Cardiology and Vascular Medicine, Faculty of Medicine, Universitas Airlangga, Prof Moestopo \\ Street 6-8, Surabaya 60286, Indonesia
}

\begin{abstract}
Pheochromocytoma is a catecholamine-producing tumour that poses diagnostic problems because its clinical presentation often mimic certain diseases, including cardiovascular disorders. It is a rare disease. The effects of excessive catecholamine secretion cause a variety of cardiovascular presentation in pheochromocytoma, ranging from the most common, namely hypertension, to life-threatening case such as hypertensive emergency, shock, supraventricular or ventricular arrhythmias, pulmonary edema, and acute coronary syndromes. The principal medical treatment for pheochromocytoma is blockade of adrenergic receptors. However, surgical or tumour resection often provides complete resolution of abnormal myocardial dysfunction or arrhythmias. As clinicians, we must be aware of the characteristics of the cardiovascular manifestations of pheochromocytoma so that we can make an earlier diagnosis and more appropriate management.
\end{abstract}

Keywords: Pheochromocytoma, Cardiovascular manifestations, Endocrine Heart Disease

Keywords: Pheochromocytoma, Cardiovascular manifestations, Endocrine Heart Disease Introduction

\section{Introduction}

Pheochromocytoma is etymologically derived from the Greek. "Phios" means black, "Chroma" means colour and "Cytoma" means tumour. Pheochromocytoma refers to the blackish-brown colour of tumour cells when stained with chromium salts (Pourian et al, 2016). These tumours usually originate from the adrenal glands, with a triad of clinical symptoms, namely headache, palpitations, diaphoresis accompanied by paroxysmal hypertension or unstable blood pressure (Delekta et al, 2015). Pheochromocytoma is rare, with an estimated prevalence in hypertensive patients of $0.1 \%-0.6 \%$ and an incidence of new cases of 2-8 cases per 1 million people. These tumours are often benign, with a malignancy prevalence of only $10 \%$ of all patients with pheochromocytoma. The median age at diagnosis is 40 years (Vilcant et al, 2017).

Pheochromocytoma is a functional tumour derived from chromaffin cells of the adrenal medulla and paragangliomas. Chromaffin cells secrete catecholamines, such as the hormones adrenaline (epinephrine), norepinephrine, and dopamine that stimulate high blood pressure. The most abundant catecholamine in the normal adrenal gland is epinephrine. Most pheochromocytomas secrete mainly norepinephrine, and only about $15 \%$ secrete epinephrine [1]. In $20 \%$ of cases, chromaffin cells grow outside their normal locations in the adrenal glands, such as in the organs of Zuckerkandl (75\%), thorax, mediastinum, abdomen, and pelvis. $70 \%$ of 
pheochromocytomas that grow outside the adrenal glands and 5\% that grow inside the adrenal glands are malignant. The enzyme dopamine beta-hydroxylase, which converts dopamine to norepinephrine, is absent in immature tumours. This condition is why dopamine-secreting tumours have a higher probability of malignancy (Pourian et al, 2016).

Many factors can cause pheochromocytoma. In most cases, genetic and environmental factors play a significant role. 25-33\% of pheochromocytomas are due to familial factors. Mutations in the VHL, RET, NF1, SDHB and SDHD genes are all known to cause familial pheochromocytoma or extra-adrenal paraganglioma (Vilcant et al, 2017).

\section{General Clinical Presentation and Principal Management}

The clinical presentation varies so much that pheochromocytoma is called "The Great Masquerader". The typical classic triad of symptoms are palpitations, headache and diaphoresis. The presence of these three clinical manifestations accompanied by hypertension often leads to the diagnosis of pheochromocytoma. However, pheochromocytoma can be asymptomatic for many years, and some tumours grow to a certain size that only causes symptoms in the patient. The dominant sign is hypertension (Pourian et al, 2016).

In addition to the classic triad, other symptoms that patients with pheochromocytoma often complain of are anxiety, tightness, chest pain, abdominal or low back pain, nausea and vomiting, tremors, flushing, dizziness, blurred eyes, and paresthesias (Pourian et al, 2016). A catecholamine crisis can also lead to heart failure, pulmonary edema, arrhythmias, and intracranial haemorrhage (Neuman et al, 2915). When we suspect a diagnosis of pheochromocytoma, we should make the diagnosis by measuring urine or plasma metanephrine levels, as well as imaging studies such as CT and MRI.

The mainstay of treatment for pheochromocytoma is definitive surgery such as total or partial adrenalectomy. However, specific therapy for each cardiovascular manifestation, especially emergencies, is essential for lifesaving efforts while waiting for definitive surgery preparation. In pheochromocytoma surgery, it is important to maintain a normal adrenal cortex, especially in hereditary disorders where bilateral pheochromocytoma is common. Preoperative preparation is crucial, including blood pressure maintained below 160/90 before surgery. Prazosin, terazosin, and doxazosin can be used as alternatives to short-acting alpha-blockers. Cardioselective beta-blockers such as metoprolol and atenolol can be used once adequate alpha-blocker effects have been achieved. Antihypertensives such as calcium channel blockers or ACE inhibitors can also be used effectively (Pourian et al, 2016).

Minimally invasive techniques (laparoscopy or retroperineoscopy) have become the standard approach to pheochromocytoma surgery due to fewer complications, faster healing, and optimal cosmetic results. Pheochromocytomas outside the adrenal glands, such as those in the thorax, can also be surgically treated endoscopically (Neuman et al, 2015).

\section{Cardiovascular Presentation of Pheochromocytoma}

Cardiovascular manifestation in pheochromocytoma range from mild to life-threatening emergencies due to the effects of catecholamine excess (Prejbisz et al, 2011). (Figure 1).

\subsection{Hypertension in Pheochromocytoma}

Approximately $90 \%$ of patients with pheochromocytoma have paroxysmal hypertension or sustained hypertension (Gu et al., 2017). Hypertension in pheochromocytoma is usually characterized by high peripheral resistance and a low cardiac index. Noradrenaline increases peripheral vascular resistance, which increases systolic blood pressure (SBP) and diastolic blood pressure (DBP). While adrenaline increases cardiac output and SBP, it does not affect DBP due to its stimulatory effect on two adrenergic receptors causing peripheral vasodilation. In adrenaline-secreting pheochromocytoma, episodic symptoms that often appear are palpitations, 
headache, syncope, anxiety, and hyperglycemia due to over-stimulation of receptors. Meanwhile, noradrenalineproducing tumours are often characterized by sustained hypertension due to the effects of excess catecholamines that stimulate alpha receptors. Tumours that predominantly secrete dopamine often resulted in normotension or even hypotension (Prejbisz et al, 2011)

Sustained hypertension is usually associated with structural abnormalities of the arteries, but it is still debated whether structural changes in blood vessels cause hypertension or vice versa. An increase in the media-lumen ratio results from vascular remodelling. Endothelial function abnormalities are also found in patients with pheochromocytoma, although this is also present in other causes of secondary hypertension (Prejbisz et al, 2011).

Drug of choice in management of hypertension in phaechromocytoma is alpha-adrenergic blockers, especially phenoxybenzamine or doxazosin. Clinician also can add other drugs such as calcium channel blocker if needed. We should never used beta blockers before start alpha-adrenergic blockade agent to avoid unopposed alpha-adrenergic receptor stimulation ( $\mathrm{Gu}$ et al, 2017).

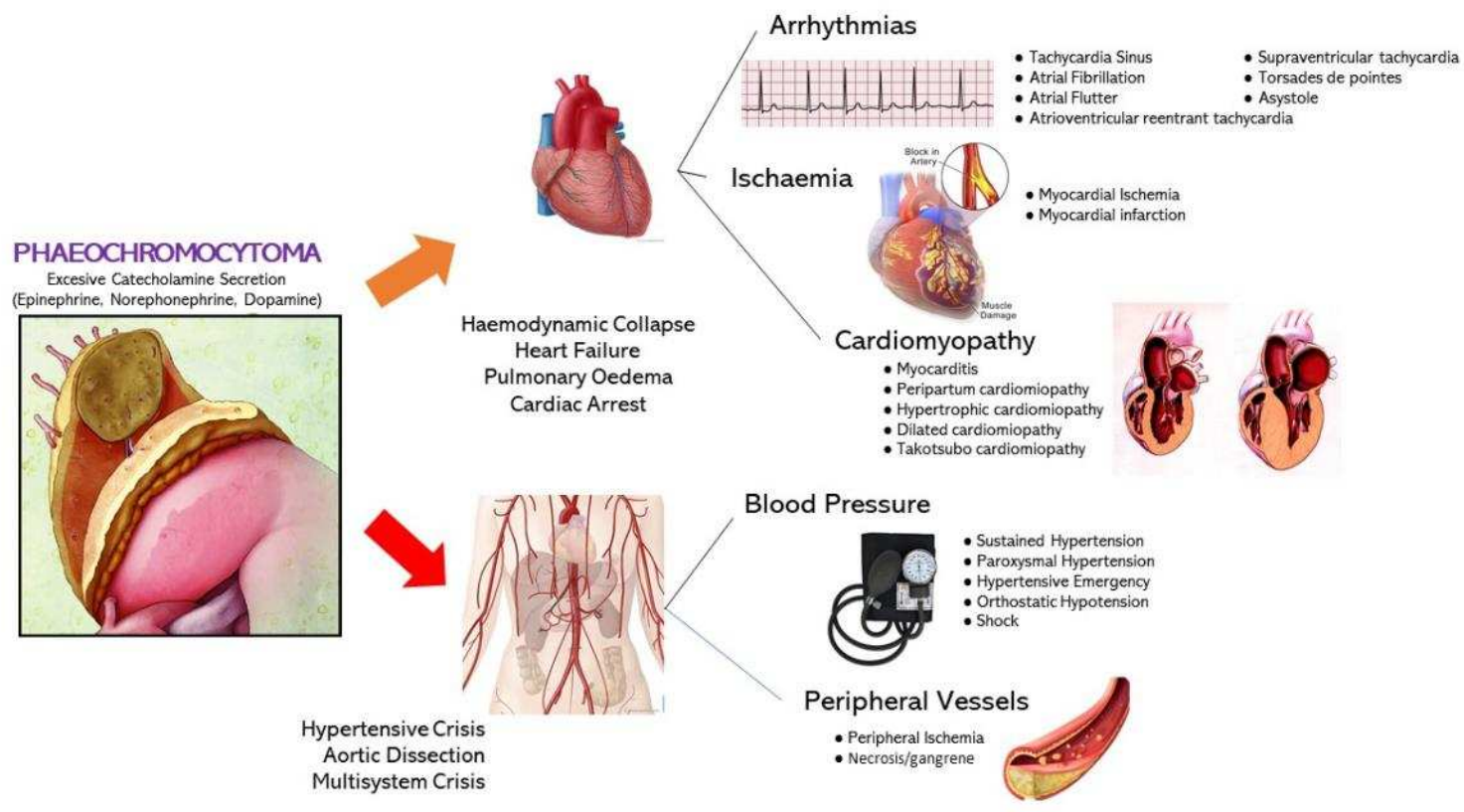

Figure 1.1 Cardiovascular Presentations of Phaeochromocytoma

\subsection{Orthostatic Hypotension in Pheochromocytoma}

Orthostatic hypotension often accompanies persistent hypertension, occurring in $70 \%$ of patients with pheochromocytoma ( $\mathrm{Gu}$ et al, 2017). Orthostatic hypotension occurring in untreated hypertensive patients should be considered as a diagnostic clue of pheochromocytoma. Most are asymptomatic and rarely have orthostatic hypotension leading to syncope. Although hypotension usually occurs only in patients with a dominant adrenaline or dopamine secretion pheochromocytoma, it can also occur in those with noradrenaline secretion (Shawa et al, 2014). 
The mechanism of orthostatic hypotension is unknown, but several possibilities exist, including hypovolemia, impaired vasoconstrictor response to catecholamines, desensitization of alpha-adrenergic receptors, and feedback inhibition of sympathetic noradrenaline released either by sympathetic inhibition or as a result of stimulation of presynaptic alpha-2 adrenergic receptors (Prejbisz et al, 2011).

Management of orthostatic hypotension in patients with pheochromocytoma should focus on efforts to restore volume. Steroid administration is often associated with fatal side effects, so there is no indication for mineralocorticoid administration in pheochromocytoma patients with orthostatic hypotension (Shawa et al, 2014).

Restoration of blood volume before surgical therapy must be maintained so that there is no decrease in blood volume leading to hypotension and shock. Pheochromocytoma should be suspected when the vascular collapse is unexplained and is associated with significant abdominal pain, pulmonary edema, mydriasis unresponsive to light stimulation, diaphoresis, cyanosis, hyperglycemia, and leukocytosis. Patients with acute myocardial infarction or acute cardiomyopathy may also present with hypotension, but this is usually recognizable. Shock in patients with pheochromocytoma can also be triggered by certain drugs such as dopamine receptor antagonists, tricyclics, and steroid hormones (Shawa et al, 2014).

\subsection{Arrhytmia in Pheochromocytoma}

In patients with pheochromocytoma, excessive amounts of catecholamines can stimulate beta-adrenergic receptors and trigger mild or severe arrhythmias. However, many factors will trigger this condition. In both humans and mice with pheochromocytoma, prolonged catecholamine stimulation decreased receptor density and induced cardiovascular adrenergic receptor desensitization. Reflex bradycardia and nodal escape rhythms have been reported, not only during hypertensive emergencies but also during episodes of increased vagal tone in patients with pheochromocytoma (Prejbisz et al, 2011). 50-70\% of patients with pheochromocytoma complain of palpitations. Although sinus tachycardia is the most common, pheochromocytoma can produce a wide variety of arrhythmias, including atrial fibrillation or flutter, supraventricular tachycardia, ventricular fibrillation, torsades de pointes, atrioventricular reentrant tachycardia, and asystolic arrest (Gu et al, 2017). Patients with paroxysmal or recurrent arrhythmias accompanied by diaphoresis, hypertension, anxiety or pallor, should be suspected of having a diagnosis of pheochromocytoma (Shawa et al, 2014).

Intravenous esmolol, a short-acting cardioselective beta-blocker, can be used to control the rate of atrial fibrillation or atrial flutter at a dose of $0.5 \mathrm{mg} / \mathrm{kg}$ intravenously over 1 minute followed by an intravenous infusion of $0.1-0.3 \mathrm{mg} / \mathrm{kg}$ per minute). However, it is necessary to give an alpha-blocking agent before using a beta-blocker so that there is no excessive stimulation of alpha receptors resulting in a hypertensive emergency (Prejbisz et al, 2011).

\subsection{Myocardial Ischemia and Myocardial Infarction in Pheochromocytoma}

Only a minority of patients with pheochromocytoma have symptoms related to myocardial ischemia or myocardial infarction. Recent clinical studies have found an association between pheochromocytoma and myocardial infarction. In some cases, myocardial damage is documented on electrocardiogram (ECG), echocardiography, and angiography. In cases for which angiographic data were obtained, less than half of patients had significant coronary atherosclerosis and classic coronary risk factors. In these patients, the excess amount of catecholamines secreted by the tumour exacerbates the course of myocardial infarction. Meanwhile, in cases without significant coronary atherosclerosis, myocardial infarction in patients with pheochromocytoma is caused by the direct toxic effects of catecholamines which cause necrosis and fibrosis of myocardial cells. High catecholamine levels cause ischemia by increasing myocardial oxygen consumption, disrupting the hemodynamic balance of myocardial supply and demand and rising afterload (Vilcant et al, 2017). 
Patients can present with chest pain, tachycardia, diaphoresis, anxiety which accompanied by an ECG appearance of $\mathrm{T}$ waves, hyperacute $\mathrm{T}$, diffuse low voltage, and other nonspecific ECG changes. One essential ECG change in pheochromocytoma is repolarization ECG changes which associated with QT interval prolongation (Y-Hassan et al, 2020). It happened due to the effects of catecholamines that stimulate coronary artery vasoconstriction and simultaneously increase myocardial oxygen demand through stimulation of heart rate and cardiac contractility, resulting in ECG abnormalities without coronary atherosclerosis. However, clinician could find cardiac marker elevation ( $\mathrm{Gu}$ et al, 2017). The repolarization ECG changes, moderate elevated cardiac markers and usually normal coronary angiography are common findings (Y-Hassan et al, 2020).

\subsection{Cardiomyopathy and Myocardial Hypertrophy in Pheochromocytoma}

Phaeochromocytoma can cause cardiomyopathies, such as hypertropic, dilated, takotsubo and peripartum. Several mechanisms may explain the acute and chronic myocardial damage associated with catecholamines. Catecholamines can directly affect the myocardium by increasing lipid mobility, calcium overload, free radical production, or increasing sarcolemmal permeability. Myocardial damage can occur secondary to a persistent increase in cardiac oxygen demand along with decreased oxygen availability due to the effects of catecholamines that trigger coronary vasoconstriction and platelet aggregation (Prejbisz et al, 2011).

High levels of catecholamines in the blood released by tumours can trigger myocarditis and cardiomyopathy. Myocarditis has previously been reported in patients with pheochromocytoma. In a case report of 26 patients at the Mayo Clinic who died of pheochromocytoma complications, $58 \%$ were diagnosed with active myocarditis. The pathological changes of active myocarditis are similar to the lesions found in the myocardium of animals treated with catecholamine injections in several laboratories (Gu et al, 2017).

High levels of catecholamines in the blood can cause dilated or hypertrophic cardiomyopathy. However, dilated cardiomyopathy is more common. Patients with pheochromocytoma and heart failure have atypical symptoms that are difficult to distinguish from idiopathic dilated cardiomyopathy. Patients with advanced dilated cardiomyopathy who will undergo heart transplantation should be excluded from causes of reversible cardiomyopathy, including pheochromocytoma. Several patients who had undergone successful heart transplantation then later diagnosed with pheochromocytoma have indistinguishable clinical symptoms compared to dilated cardiomyopathy (Bernard et al, 2012).

"Takotsubo" cardiomyopathy is a condition of patients with signs and symptoms of acute myocardial infarction without coronary artery stenosis or spasm with the appearance of the heart in the form of a Japanese octopus fishing pot, called "Takotsubo". The rounded apex of the heart indicates the effect of local toxic concentrations of catecholamines in the absence of coronary artery disease. High concentrations of noradrenaline stimulate basal hyperkinesis, increase mechanical stress on the apex wall and end-diastolic pressure. Another patient with pheochromocytoma-induced cardiomyopathy has severe left ventricular dysfunction, basal segment akinetic, midventricular and apical hyperkinetic. This percentage is called "Inverted Takotsubo cardiomyopathy". Therefore, there is no definite pattern of ventricular dysfunction in patients with pheochromocytoma-induced Takotsubo cardiomyopathy (Prejbisz et al, 2011). Treatment of pheochromocytoma-induced-cardiomyopathy is primarily supportive care until definitive surgical therapy can be performed (Gu et al, 2017).

\subsection{Peripheral Ischemia in Pheochromocytoma}

Although very rare, pheochromocytoma can result in peripheral ischemia that results in necrosis or gangrene. This complication occurs as a result of severe vasoconstriction or diffuse arterial vasospasm induced by hypercatecholaminemia. Ischemia can also occur due to arterial occlusion of cardiac thrombus embolism in patients with catecholamine-induced arrhythmias, but this is extremely rare (Prejbisz et al, 2011). Patient can 
have a similar presentation with peripheral artery disease, complaining with claudication or limp pallor (Gu et al, 2017).

\section{Life-Threatening Cardiac Manifestations}

In some cases of pheochromocytoma, the cardiac presentation that appears can be life-threatening and cause death. The most common life-threatening cardiac manifestation of pheochromocytoma is a hypertensive emergency due to the rapid and excessive release of catecholamines from the tumour. Less common are malignant arrhythmias, shock, aortic dissection and acute heart failure due to myocardial dysfunction (Leite et al, 2010)

\subsection{Hypertensive Emergency in Pheochromocytoma}

An increase in systolic blood pressure in patients with pheochromocytoma can reach a very high and dangerous value above $200 \mathrm{mmHg}$. This situation becomes a life-threatening hypertensive emergency when accompanied by acute target organ damage. Hypertensive emergencies can also result from using certain drugs in patients with pheochromocytoma, such as beta-blockers that are not accompanied by adequate use of alphablockers. Hypertensive crisis can happen in $75 \%$ of patients with pheochromocytoma as often as once weekly. Symptoms of a hypertensive crisis may vary, such as headache, confusion, visual disturbances or tachycardia. This condition can lead to target organ damage such as acute myocardial infarction, congestive heart failure or cerebrovascular disorders (Gu et al, 2017).

Intravenous phentolamine is the treatment choice for a hypertensive emergency in patients with pheochromocytoma. The drug is usually given in an intravenous bolus dose of $2.5-5 \mathrm{mg}$ at a rate of $1 \mathrm{mg} / \mathrm{min}$. This dose should be repeated every 3-5 minutes until hypertension is controlled because of the short half-life of phentolamine. Phentolamine can also be given by continuous infusion. However, due to the availability of other drugs with safer pharmacokinetic profiles, phentolamine is no longer used as a hypertensive emergency treatment during surgical anaesthesia. Intravenous vasodilators such as sodium nitroprusside, nicardipine, fenoldopam, and nitroglycerin provide more effective short-term control in cases of intraoperative hypertension. It is easier to titrate, has a shorter duration of action and can be used alone or with other vasodilators. Another alternative therapy is urapidil, a selective alphal adrenergic receptor antagonist that can be given as an intravenous bolus dose (25-50 mg) or accompanied by an infusion (50-100 mg/kg per minute) (Čtvrtlík et al, 2018).

\subsection{Malignant Arrhytmia in Pheochromocytoma}

Although rare, pheochromocytoma can result in QT prolongation, even malignant arrhythmias such as ventricular tachycardia. Several recent case reports have described cases of ventricular tachycardia accompanying a patient with suspected acute myocardial infarction but no coronary artery stenosis on angiography. The more common arrhythmias in pheochromocytoma are sinus tachycardia, atrial fibrillation and atrial flutter (Prejbisz et al, 2011).

Several cases of pheochromocytoma have complications of malignant arrhythmias, namely life-threatening ventricular tachycardia, such as a young woman from Beijing who complained of the classic triad of pheochromocytoma with recurrent ventricular tachycardia in the course of the disease (Li et al, 2016). Paulin et al. suggested that the sudden release of catecholamines from pheochromocytoma is related to VT's mechanism (Paulin et al, 2009). Excess levels of catecholamines cause abnormal electrical activity of the myocardium, such as the excessive opening of ion channels, increased function of the ion exchange pump, which causes large amounts of sodium, potassium and calcium to enter through the membrane resulting in automatic, intensive myocardial conductivity. In addition, excess catecholamines can reduce the threshold for ventricular fibrillation, 
resulting in sudden death or abnormal repolarization that underlies various types of rapid arrhythmias (Zhang et al, 2016).

Sustained ventricular tachycardia in pheochromocytoma can be treated by ablation, but such therapy cannot stop the systemic arrhythmogenic mechanisms due to the effects of catecholamines, so tachyarrhythmias will often recur. A Pheochromocytoma should be considered as a diagnosis in a hypertensive patient with a typical clinical presentation of palpitations and headaches with an ECG showing VT or other tachyarrhythmias. Biochemical and imaging tests will guide the diagnosis, and symptoms often have a complete resolution after tumour surgery. Preoperative use of phentolamine may be considered. Beta-blockers, such as propranolol, can be used in catecholamine-induced tachyarrhythmias but should be started after adequate doses of alpha-blockers administration to prevent hypertensive emergencies (Zhang et al, 2016).

Sinus arrest due to catecholamine release, often accompanied by nodal escape rhythms, has been reported in several case reports. On the other hand, atrioventricular dissociation associated with pheochromocytoma only occurred in 2 cases. Unfortunately, the diagnosis of pheochromocytoma leading to sinus arrest, AV dissociation, supraventricular and ventricular arrhythmias are often delayed, and the patient has had a pacemaker inserted and even ablated his bundle. Tumour resection therapy can solve all cases without the need for a pacemaker or ablation (Zhang et al, 2016).

\subsection{Shock in Pheochromocytoma}

Sudden cessation of catecholamine secretion in patients with inadequate circulating volume, and desensitization of adrenoreceptors due to prolonged exposure to catecholamine-induced hypertension, are the main mechanisms that explain shock in patients with pheochromocytoma (Shawa et al, 2014). Hemorrhagic necrosis of catecholamine-producing tumours is also associated with severe shock. Another less well-known mechanism is shock due to the negative inotropic effect of hypocalcemia. Shock in patients with pheochromocytoma is often responsive to volume addition (Prejbisz et al, 2011).

\subsection{Acute Heart Failure in Pheochromocytoma}

Acute heart failure or pulmonary edema is a rare percentage of pheochromocytoma. However, in the vast majority of cases, pulmonary edema is of cardiac origin. Catecholamines trigger a transient increase in pulmonary capillary pressure and increase pulmonary neutrophil accumulation (Prejbisz et al, 2011).

\subsection{Aorta Dissection in Pheochromocytoma}

Acute dissection of the abdominal and thoracic aorta is a rare but life-threatening disorder of pheochromocytoma. Only a few cases report this incident. Pheochromocytoma may be found during evaluating a patient with aortic dissection or during emergency surgery of an abdominal aortic aneurysm. Phentolamine as alpha-blocking agent should be given during emergent surgery (Gu et al, 2017).

\section{Conclusion}

Cardiovascular presentation of pheochromocytoma vary widely, ranging from mild to life-threatening abnormalities requiring immediate action, such as hypertensive emergencies, malignant arrhythmias including ventricular tachycardia and ventricular fibrillation, shock, and acute heart failure or pulmonary edema. Therefore, it is necessary to consider the diagnosis pheochromocytoma in patients with cardiovascular presentations accompanied by unstable blood pressure and/or the classic triad of symptoms, namely headache, palpitations, and diaphoresis.

\section{Conflict of Interest}

The authors declare no conflict of interest. 


\section{References}

Bernard, M., Gold, M., Cuoco, F. 2012. Pheochromocytoma Presenting as Recurrent Stress Cardiomyopathy with Multiple Monomorphic Ventricular Tachycardias. The Journal of Innovations in Cardiac Rhytm Management, 3: 803-808

Delekta, J., Riadhi, S., Eschen, O. 2015. Rare cause of ventricular tachycardia: Pheochromocytoma. Journal of Cardiology Cases, 11: 6265

Gu et al. 2017. Cardiovascular Manifestations of Pheochromocytoma. Cardiology in Review, 25 (5): 215-222

Leite, L., Macedo, P., Santos, S., Quaglia, L., Mesaz, C., Paola, A. 2010. Life Threatening Cardiac Manifestations of Pheochromocytoma. Case Reports in Medicine 2010: 1-4

Li, S., Wang, T., Wang, L., Pang, Z., Ma, B., Li, Y., Dong, H. 2016. Ventricular Tachycardia and Resembling Acute Coronary Syndrome during Pheochromocytoma Crisis. Medicine, 95 (14): 3297-3301

Neuman, H. 2015. Pheochromocytoma. Editors: Kasper D, Haver S, Jameson J, Faucy A. 19th edition. New York: Mc Grawn Hill Education, pp: 2329-2335.

Paulin, F., Klein, G., Gula, L., Skanes, A., Yee, R., Krahn, A. 2009. QT Prolongation and Monomorphic VT Caused by Pheochromocytoma. Journal of Cardiovascular Electrophysiology, 20 (8): 931-934

Pourian, M., Mostafazadeh, D., Soltani, A. 2016. Does this Patient Have Pheochromocytoma? A systematic Review of Clinical Signs and Symptoms. Journal of Diabetes and Metabolic Disorders, 15 (11): 1-12

Prejbisz, A., Lenders, J., Eisenhofer, G., Januszewicz, A. 2011. Cardiovascular Manifestations of Pheochromocytoma. Journal of Hypertension, 29: 2049-2060.

Shawa, H., Bajaj, M., Cunningham, G. 2014. Pheochromocytoma-induced Atrial Tachycardia Leading to Cardiogenic Shock and Cardiac Arrest. Texas Heart Institute Journal, 41 (6): 660-663.

Vilcant, V. 2017. Pheocromocytoma-induceed Cardiomyopathy Mimicking Acute Coronary Syndrome. The Journal of The American Osteopathic Association, 17: 537-540

Y-Hassan S., Falhammar H. 2020. Cardiovascular Manifestations and Complications of Pheochromocytomas and Paragangliomas. Journal of Clinical Medicine, 9 (2435) : 1-19.

Zhang, M., Mao, W., Wu, D., Liu, P. 2016. Pheochromocytoma with Ventricular Tachycardia as The Presenting Symptoms. Chinese Medical Journal, 129 (12): 1505-1506 